\title{
Personal Pollen Sampler
}

\section{Pollenbelastung messen statt schätzen}

\author{
Auf dem diesjährigen Meeting der American Academy of Allergy, \\ Asthma and Immunology wurde der "persönliche Pollenzähler" \\ präsentiert. Das System hat die Firma Bluestone Technology gemeinsam \\ mit der FH Mainz, der Berliner Charité und der health \& media GmbH \\ entwickelt. Was es genau mit dem persönlichen Pollenzähler auf sich \\ hat, erklärt der produktverantwortliche Dipl.-Ing. Torsten Sehlinger.
}

? Was muss man sich unter einem „persönlichen Pollenzähler" vorstellen?

Sehlinger: Das System besteht aus zwei Komponenten: Die erste ist ein tragbares Gerät, mit dem die individuelle Pollenexposition des Trägers über einen längeren Zeitraum exakt bestimmt werden kann. Neben Pollenart und -menge bestimmt es den Standort, misst Luftfeuchtigkeit, Temperatur, Luftdruck und UV-A- und UV-BStrahlung. Die zweite Komponente betrifft die Auswertung der Pollenmessung. Um die Praktikabilität zu gewährleisten, haben wir diesen Prozess teilautomatisiert.

\section{? Wie funktioniert das System?}

Sehlinger: Für aussagekräftige Ergebnisse muss der Nutzer das Gerät während der Untersuchungsphase immer mit sich führen. Da es $90 \mathrm{~g}$ leicht und nur $2,2 \mathrm{~cm}$ lang, $2,2 \mathrm{~cm}$ breit und $12 \mathrm{~cm}$ hoch ist, dürfte es kaum stören. Vor Inbetriebnahme muss der Nutzer zunächst eine Kassette einlegen. Die Kassette sammelt Pollen, Sporen und Staub aus der Umgebungsluft. Sie ist nach zwei oder drei Tagen zu wechseln und kann beim Arzt abgegeben oder direkt an uns geschickt werden. Die gespeicherten Zusatzinformationen werden elektronisch übermittelt: Der Nutzer schließt das Gerät an seinen Rechner an und lädt die Daten auf den Zentralrechner oder transferiert die Daten via Bluetooth mit seinem Mobiltelefon. Während der Messphase sollte der Patient alle Symptome notieren, etwa mit seinem Smartphone über das Pollentagebuch des Polleninformationsdienstes oder andere dafür entwickelte Apps. So lassen sich den Beschwerden der Ort der Exposition sowie Art und Menge der Pollen zuordnen.
? Wozu braucht man den Pollenzähler? Sehlinger: Ausschlaggebend für die Entwicklung war, dass die anhand des Wohnortes durch Interpolation berechnete Pollenbelastung sehr ungenau ist. Das erschwert die Beurteilung der Wirksamkeit von Immuntherapien und Präparaten zur Symptomkontrolle in klinischen Studien. Zwar gibt es in Deutschland vergleichsweise viele Pollenfallen, dennoch sind bei einer durchschnittlichen Distanz von $100 \mathrm{~km}$ auch hierzulande die Interpolationen extrem ungenau, zumal die Fallen bundesweit nicht gleichmäßig verteilt sind. In Rheinland-Pfalz und im Saarland gibt es keine einzige. Die Berechnungen berücksichtigen auch nicht, ob der Betroffene in der Stadt oder auf dem Land wohnt, ob er sich viel draußen aufhält oder in geschlossenen Räumen.

? Für wen ist das System gedacht?

Sehlinger: Die ursprüngliche Idee ist, Probanden klinischer Studien mit einem Pollenzähler auszustatten. Damit fällt die Fehlerquelle der Interpolation weg, und die Patientenzahl, die für ein statistisch aussagekräftiges Ergebnis erforderlich ist, kann gesenkt werden.

\section{? Gibt es weitere Anwendungen?}

Sehlinger: Niedergelassene Ärzte profitieren ebenfalls: Das System erleichtert die Anamnese. Patienten fällt die zeitliche Zuordnung ihrer Symptome oder deren Zusammenhänge zu Umweltfaktoren oft schwer, entsprechend vage sind ihre Ausführungen. Der Pollenzähler weist objektiv nach, welchen Pollen der Patient ausgesetzt war, und ermöglicht eine zielgenauere Allergietestung. Vorstellbar ist auch, dass Privatpersonen das System nutzen, um ihre

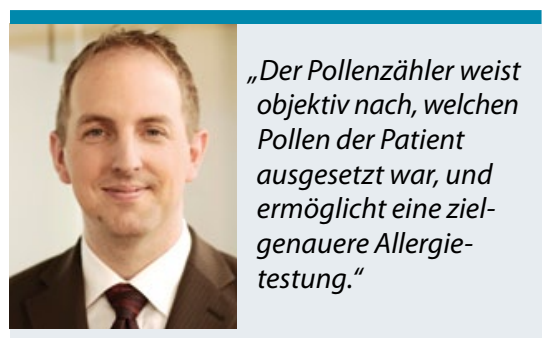

Dipl.-Ing. Torsten Sehlinger,

Bluestone Technology $\mathrm{GmbH}$

Symptome besser vorhersagen und die nötige Antihistaminmenge exakter abschätzen zu können.

? Ist der persönliche Pollenzähler bereits im Einsatz?

Sehlinger: Die erste Patientenstudie beginnt in sechs Wochen. Wir werden etwa 30 Patienten der Berliner Charité und der Medizinischen Universität Wien mit diesen Geräten ausstatten. Da wir bislang nur die Effektivität getestet haben, wird sich nun zeigen, wie anwenderfreundlich das Gerät tatsächlich ist. Anhand der gesammelten Daten überprüfen wir zudem, inwieweit die individuell gemessene Belastung mit den interpolierten Werten korreliert, und welche Faktoren bei der Interpolation berücksichtigt werden müssen, um bessere Näherungswerte zu erhalten.

? Ab wann ist das System marktfähig? Sehlinger: Für den Einsatz in klinischen Studien wird das System ab Juni/Juli 2013 zum Preis von $300 €$ erhältlich sein. Für den Consumer- und NiedergelassenenBereich sind die Analysekapazitäten der limitierende Faktor. Die Herausforderung ist, die bereits teilautomatisierte Auswertung der Teststreifen noch effizienter zu machen. Hier sind wir auf einem gutem Weg.

! Vielen Dank für das Gespräch.

Das Interview führte Dr. Dagmar Kraus. 